\title{
APLICACIÓN DEL MÉTODO \\ DE VALORACIÓN BIOGEOGRÁFICA LANBIOEVA A DIVERSOS PAISAJES DE LA PATAGONIA CHILENA
}

\section{APPLICATION OF THE LANBIOEVA BIOGEOGRAPHICAL EVALUATION METHOD TO VARIOUS LANDSCAPES OF THE CHILEAN PATAGONIA}

Pedro José Lozano Valencia* Guillermo Meaza Rodríguez* José Antonio Cadiñanos Aguirre* Asier Lozano Fernández* Rakel Varela-Ona*

RESUMEN: El siguiente artículo presenta un trabajo de investigación donde se aplica la metodología LANBIOEVA (Landscape Biogeographical Evaluation) a varios paisajes vegetales de la Patagonia chilena. Hasta la fecha, dicho método se ha plasmado en numerosos trabajos y en diferentes territorios templados y boreales de Europa: Península Ibérica, Balcanes, Península Escandinava, Holanda, mediterráneos chilenos, tropicales brasileños y nicaragüenses e incluso africanos (Marruecos). Sin embargo, estos paisajes inventariados, analizados y valorados según la metodología citada cuentan con pocos estudios similares $y$, por lo tanto, muestran un gran interés. El objetivo es doble, por una parte, constatar su eficacia y viabilidad en otros ámbitos y, por otra, servir de herramienta valorativa para el diagnóstico de la calidad del medio con fines fundamentalmente conservacionistas. Con ello, el gestor puede contar con un nutrido grupo de criterios y valores que le ayuden para su correcta y sostenible gestión. Los resultados muestran que existen diferencias importantes entre unas unidades y otras. Paisajes como los boques mixtos de cohigual-lengar, el de ñirre o los matorrales de notro y mata guanaco cuentan con buenos valores mientras que otros, como algunas turberas y el matorral de mata verde, muestran registros más modestos. En general, los valores son relativamente altos pero inferiores a los registrados en otros ámbitos como el mediterráneo chileno y el atlántico de la Península ibérica. Los datos obtenidos pueden ser relevantes para la protección, ordenación o correcta gestión de los paisajes y territorios analizados.

Palabras clave: Valoración biogeográfica, INCON, PRICON, paisajes, Espacios Naturales Protegidos y Patagonia chilena.

Recibido: 19.06.20 Aceptado: 19.09.20

*Departamento de Geografía, Prehistoria y Arqueología, Universidad del País Vasco/Euskal Herriko Unibertsitatea pedrojose.lozano@ehu.eus https:orcid.org/0000-0002-1345-5704; guillermo. meatza@gmail.com https:orcid.org/0000-0003-0705-2631; joseantonio.cadinanos@ehu.eus https:orcid.org/0000-0001-7232-0722; lozanoasier6@gmail.com https:orcid.org/0000-0002-3013-4929; rakel.varela@ehu.eus https:orcid.org/0000-0001-5079-0627 


\begin{abstract}
This article presents a research paper in which the LANBIOEVA (Landscape Biogeographical Evaluation) methodology is applied to several vegetational landscapes of Chilean Patagonia. To date, this method has been applied in numerous works and in different temperate and boreal territories of Europe, such as: Iberian Peninsula, Balkans, Scandinavian Peninsula, Holland, Chilean Mediterranean, Brazilian and Nicaraguan tropical and even African landscapes (Morocco). Nevertheless, these landscapes, inventoried, analyzed and evaluated according to the above-mentioned methodology, have few similar studies and are therefore of great interest. The objective is twofold: on the one hand, to verify their effectiveness and feasibility in other areas and, on the other hand, to serve as a valuation tool for the diagnosis of the quality of the environment for conservation purposes. With this, the managers can count on a large group of criteria and values that will help them for its correct and sustainable management of the environment. The results clearly show that there are important differences between some units and others. Landscapes such as the mixed cohigual-lengar forests, the ñirre forest or the notro and mata guanaco thickets have good values, while others such as some peatlands and mata verde thicket show more modest records. In general, the values are relatively high but lower than those recorded in other areas such as the Chilean Mediterranean and the Atlantic of the Iberian Peninsula. The data obtained may be relevant for the protection, planning or correct management of the landscapes and territories analyzed.
\end{abstract}

Key words: Biogeographic assessment, INCON, PRICON, landscapes, Nature Reserves and Chilean Patagonia

\title{
INTRODUCCIÓN
}

En los últimos años muchos son los esfuerzos realizados en el campo de la conservación de espacios y especies de valor ecológico. Entre las iniciativas para la conservación de espacios encontramos metoEn los últimos años se ha realizado un gran esfuerzo en el campo de la conservación de espacios y especies de valor ecológico. Entre las iniciativas para la conservación de espacios encontramos metodologías que tratan de ofrecer métodos científicos para analizar, diagnosticar y valorar la calidad ambiental, cultural, mesológica, etc. (Strijker, Sijtsma y Wiersma, 2000). Para ello resulta muy conviene trabajar desde la planificación, ordenación y gestión de los mismos con una visión transversal que reúna cuestiones que tengan en cuenta tanto valores naturales intrínsecos como de las unidades de paisaje que van a ser valoradas y también cuestiones relacionadas con los procesos ecológicos: generación de condiciones microclimáticas adecuadas, prevención de riesgos ambientales, conservación del suelo y aminoración de los procesos erosivos, mantenimiento de comunidades biológicas interesantes; cuestiones culturales y de manejo: mantenimiento de prácticas agropecuarias extensivas y sostenibles, existencia de yacimientos arqueológicos, elementos etnográficos o simbólicos reseñables, prácticas culturales tradicionales o seculares, etc. Por último, la prioridad de conservación se encarga de asumir el nivel de amenaza predecible para 
las unidades estudiadas y se desglosa en la presión antrópica potencial a través de la demografía, la accesibilidad-transitabilidad del lugar y los peligros añadidos como fuegos más o menos recurrentes, presión inmobiliaria, residuos, etc.

De hecho, los territorios y unidades de paisaje a conservar, ordenar y gestionar además de presentar valores medioambientales, también sostienen una serie de relaciones sociales, económicas, políticas, productivas, etc., que los hacen acreedores de unas herramientas de valoración y gestión mucho más adecuadas a sus realidades y con una visión que que va más allá de los valores a conservar (Botequilha-Leitao y Ahern, 2002).

Conscientes de esta multiplicidad de campos, se diseñó un método de valoración denominado LANBIOEVA (Landscape Biogeographical Evaluation) con base en el inventario biogeográfico y en la definición de unidades de paisaje a través del estudio de su composición florística, que aúna, de forma equilibrada y exhaustiva, todas estas cuestiones de manera que se puedan realizar evaluaciones de forma parcial pero también con un resultado cuantitativo final que nos informe acerca del interés de conservación de dichas unidades. Esta metodología cuenta con una historia de cerca de 30 años y ha sido aplicada a más de 200 formaciones o paisajes vegetales a escala mundial (Europa, África y Américal, de manera que en Lozano-Valencia et al., (2020) puede verse un resumen de dicha aplicación y los resultados obtenidos, amén de la bibliografía acumulada en todos estos años. Desde principios de los 90 se buscaba un método de inventariado y valoración que fuera realmente amplio y global. Entonces, para estudios de carácter biogeográfico pero también relacionados con la ordenación del territorio, existía la metodología IRAMS que, no obstante, obviaba aspectos de raigambre natural y cultural. Por ello, ya para 1993 se comenzó a publicar los primeros resultados de la aplicación de la metodología LANBIOEVA, al principio sin referirse a espacios concretos y sin el acrónimo citado (Meaza y Ormaetxea, 1992., Meaza, 1993). Un año más tarde y, como consecuencia de una estancia de investigación, se realizó la primera aplicación a espacios y formaciones vegetales concretas, en este caso de Nicaragua (Meaza, 1994). Desde entonces se han publicado hasta un total de más de 40 trabajos (libros, capítulos de libro, artículos, ponencias y comunicaciones a congresos) sobre la aplicación de la citada metodología dentro de diferentes territorios y paisajes. En la actualidad, está siendo utilizado por investigadores y grupos de países como España, Brasil, Chile y Marruecos (Lozano, 2020).

Durante los últimos años y a partir, al menos, de más de diez proyectos de investigación financiados por gobiernos autonómicos, el gobierno central, diversas universidades y otros centros docentes y científicos se diseñó y puso en práctica, en diversos ámbitos ya citados, el presente método de valoración LANBIOEVA, de manera que se comienza a contar con un bagaje lo suficientemente amplio. Con ello se pretende la consolidación de una metodología estándar que, además, ha sido contrastada y expuesta a la crítica especializada en más de veinte congresos nacionales e internacionales diferentes y a través de artículos, libros, capítulos de libro, monografías, etc.

\section{ESTADO DE LA CUESTIÓN}

El desarrollo económico experimentado durante el siglo XX ha favorecido en buena parte la aceleración del deterioro ambiental. La pérdida de una gran cantidad de hábitats y ecosistemas naturales ha desembocado en 
un alarmante descenso de los niveles de diversidad biológica, cuyos efectos son notorios tanto a nivel global como local (Wilson, 1994). Se estima que la actividad humana ha incrementado la tasa de extinción de especies hasta situarla 1.000 veces por encima de la natural (Mc Neill, 2003). De acuerdo con estudios recientes elaborados por el Programa para el Medio Ambiente de las Naciones Unidas (PNUMA-2016), el 60\% de los ecosistemas mundiales se encuentran en estado degradado o son utilizados de manera insostenible. Asimismo, de confirmarse las previsiones acerca del calentamiento global, entre el 18 y 35\% de las especies podrían desaparecer para 2050.

Ante la progresiva pérdida de biodiversidad se han seguido distintos esquemas de actuación. Por un lado, las estrategias conservacionistas han girado en torno a la protección de determinadas especies animales y vegetales, y, por otro, han promovido la preservación de hábitats naturales mediante su declaración como espacios naturales protegidos (en adelante, ENP), regulando así los usos y actividades a desarrollar en éstos. Esta última parece configurarse como la política de conservación más adecuada puesto que el correcto estado de dichos hábitats redunda, sin duda alguna, en el mantenimiento de poblaciones viables de todas aquellas especies sensibles a su destrucción o reducción (Faith y Walker, 1996., Tscharntke et al., 2002). Las áreas protegidas son lugares en los que se trata de preservar sus recursos y paisajes. Además, existe la opinión generalizada de que la sociedad percibe estos lugares como referentes en favor de un medio ambiente saludable. Igualmente, la cuestión ambiental está adquiriendo lenta, pero gradualmente mayor presencia en las sociedades industriales avanzadas, al tiempo que en su marco de actuación pública el espacio de la política medioambiental está siendo creciente (Chan et al., 2016 ).

Los ENP han de contar con las medidas de conservación necesarias, para lo que se prevé la elaboración y puesta en marcha de planes de gestión, específicos o incluidos en otros planes de desarrollo. No podemos descartar que la protección de estos lugares, en ocasiones, pueda requerir la continuación o fomento de actividades humanas tradicionales, ya que, si bien el objetivo de la mayor parte de los códigos legales que existen a escala planetaria asumen el mantenimiento de la biodiversidad, también se tienen presentes las exigencias económicas, sociales y culturales, así como las particularidades locales de los lugares asignados (Botequilha-Leitao y Ahern, 2002). Ello debe reflejarse en los planes de gestión, que además deben considerar la evaluación a la que se ha de someter toda actividad que se ejerza en un ENP. Se entiende, por tanto, que los planes de gestión de los ENP deberían adecuar las medidas adoptadas a las actividades humanas sostenibles desarrolladas en estos lugares, así como a la potencialidad de sus impactos socioeconómicos. Trabajos como los realizados por Lozano (2001) demuestran que determinados usos tradicionales, junto a unidades ambientales culturales (por lo tanto manejadas en mayor o menor medida por el ser humanol, garantizan unos niveles de biodiversidad superiores a los registrados dentro de hábitats naturales menos transformados. 


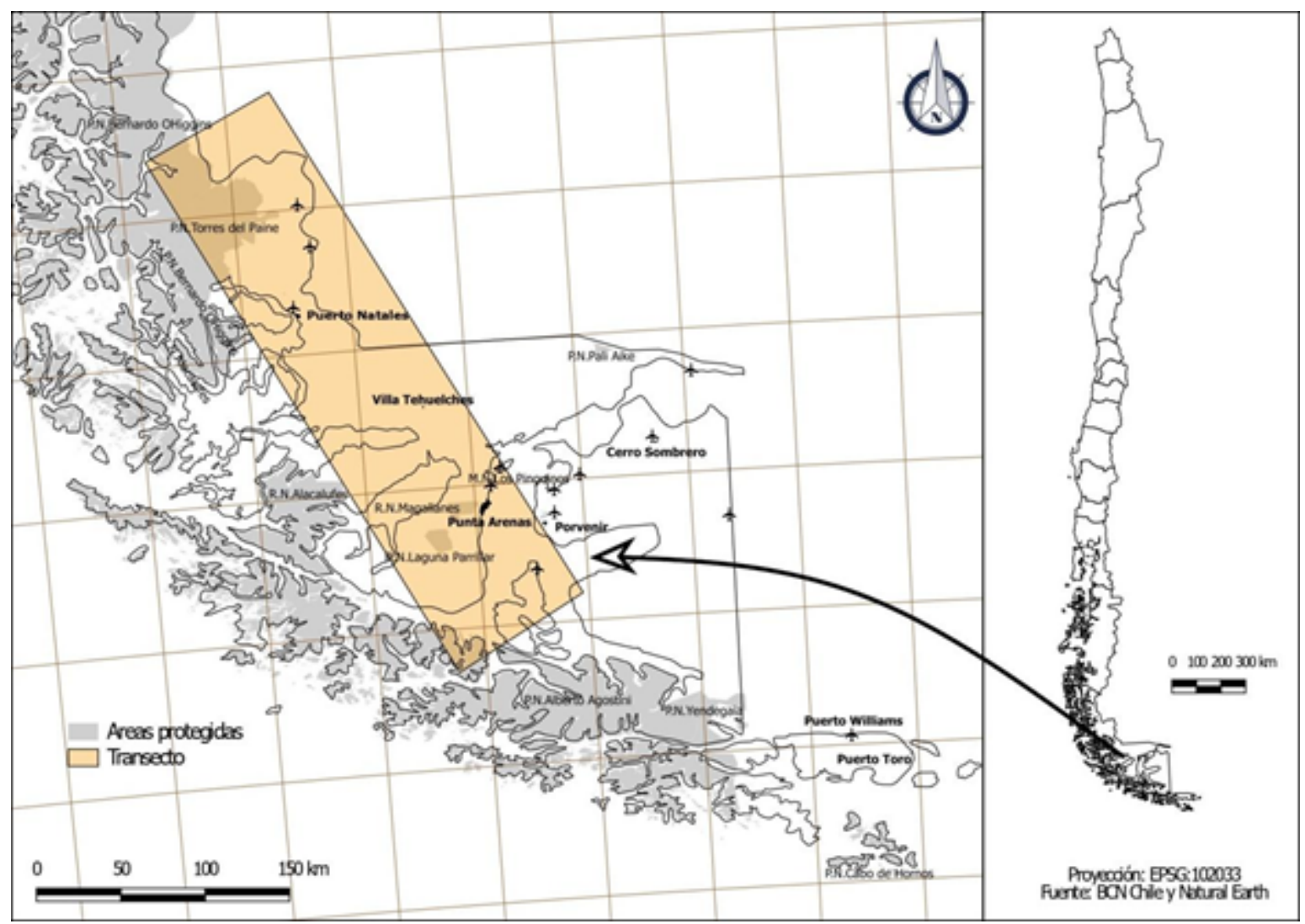

Figura 1. Mapa de localización y espacio cubierto por el transecto. Elaboración propia a partir de BCN Chile y Natural Earth.

Para ello, y como consecuencia de dos estancias en Chile, una durante el mes de noviembre de 2018 y otra durante enero de 2019, se realizó un recorrido que abarcaba desde la Península de Bruswick hasta las Torres del Paine (Figura 1). En él, y sobre todo en los ENP correspondientes, se seleccionaron diversos paisajes y ecosistemas dotados de valores naturales y culturales sobresalientes, así como con posibles problemas por presiones antrópicas de distinto tipo. En ellos es donde se ha aplicado la metodología LANBIOEVA. de diferentes paisajes y ecosistemas a escala mundial, en este caso dentro de la Patagonia chilena. Su contenido y funcionalidad práctica llevan aparejados los siguientes objetivos operativos:

- La obtención de valoraciones parciales que puedan ser tenidas en cuenta de forma sectorial atendiendo a los atributos o cuestiones que se consideren oportunas a la hora de planificar y gestionar los paisajes vegetales de la Patagonia chilena, valores naturales, culturales, mesológicos, estructurales y amenazas, entre otras.

- Asimismo, la obtención de valores generales o sintéticos que aglutinen criterios y aporten datos valorativos generales como el interés de conservación o la prioridad de
El objetivo general de este artículo es aplicar un marco metodológico integrado que sirva para la valoración y evaluación ambiental 
conservación. Tanto más en espacios nos hizo inclinarnos por llevar a cabo protegidos donde el fin último debe de ser la diferentes inventarios que abarcasen la conservación.

-La creación de un modelo metodológico de mayor cantidad unidades posibles y también las diferentes facies de éstas. Para ello, se inventariado y valoración, lo más sencillo posible, para que pueda seguir siendo aplicado a espacios y paisajes para los que no fue diseñado originalmente. En este caso, la adaptación de la metodología a la realidad del territorio patagónico y de los ecosistemas caracterizados, analizados y valorados.

\section{METODOLOGÍA}

Una de las líneas principales de la Biogeografía aplicada es la valoración, que busca analizar el estado actual del paisaje y la vegetación y realizar una evaluación cualitativa con un objetivo principalmente conservacionista. El análisis valorativo del paisaje permite, además, aglutinar especialistas de diferentes áreas de conocimiento tales como la botánica, la ecología, la geografía, la ingeniería y otras áreas técnicas y profesionales (Cadiñanos y Meaza, 1998 a y 1998b), por lo que resulta de sumo interés elaborar procedimientos que hayan sido consensuados y puedan ser asumidos por un número importante de expertos.

El primer paso fue definir las unidades de paisaje y la ubicación de las parcelas más idóneas para el inventariado y través de la fotointerpretación y el trabajo campo se llevaron a cabo los inventarios biogeográficos. Para llevar a cabo esta investigación se escogieron aquellas parcelas de vegetación que a priori contaban con las características tipo del paisaje que se pretendía estudiar. La escasez del tiempo para la investigación, tres semanas en cada una de las dos visitas, diseñó un transecto que atravesara de sur a norte la Patagonia chilena entre la zona de Punta Arenas y las Torres del Paine. Se seleccionaron parcelas repartidas por dicha franja latitudinal y que en la mayoría de los casos se encontraban en zonas que contaban con alguna figura de protección, lo que podría asegurar un buen estado de la vegetación. También se marcó un número concreto de parcelas teniendo en cuenta su representatividad. Se han llevado a cabo un total de 22 inventarios. En las parcelas elegidas se pusieron en marcha las herramientas metodológicas necesarias para el inventariado y posterior valoración, tanto sobre el terreno como posterior para cotejar los resultados de campo y para su evaluación definitiva. Se han realizado, por tanto, dos visitas en años diferentes y estaciones diferentes para evitar precisamente contingencias climáticas u otro tipo de eventos como puede ser la diferente fenología y asegurar que se inventarían la mayor cantidad de especies diferentes. Como veremos más adelante, en la Tabla 1 se pueden comprobar las características geográficas de las diferentes parcelas donde se llevaron a cabo los inventarios. Como se podrá comprobar, la mayor parte de ellas pertenecen a espacios de alto interés ambiental como son la Laguna Parrillar, Reserva Magallanes, Seno Otway, Torres del Paine, Glaciar del Grey, Cuernos del Paine,

etc.

El modelo de inventario es específico y desde su diseño inicial y tras ser aplicado en numerosas territorios se ha corregido /Cadiñanos y Meaza, 1998a y 1998b., Cadiñanos y Meaza, 2000., Cadiñanos, Meaza y Lozano, 2002., Cadiñanos et al., 
2002., Cadiñanos y Lozano, 2006., Cadiñanos, Lozano y Quintanilla, 2016., Lozano et al., 2020) con el fin de recopilar los datos geográficos y medioambientales necesarios para la valoración biogeográfica de la comunidad vegetal a estudiar. E primer paso es tomar los datos de localización e identificación del lugar (coordenadas UTM, topónimos, etc.), cuestiones geográficas y medioambientales generales ltopografía, litología, geomorfología, cuestiones edáficas, hidrológicas, etc.l, fotografías de la parcela, etc. En cuanto al propio inventario en sí, se han de catalogar todos los taxones de la flora vascular presentes, los de la flora fúngica y liquénica (hongos y líquenes), la cobertura y especies de la briófita lestrato muscinall), indicando la cobertura de cada especie -escala de 6 clases (5: máximo, +: mínimo). Esta tarea se ha de realizar en cada uno de los cuatro estratos en que se dividen convencionalmente las comunidades (estrato $>5 \mathrm{~m}$, estrato entre 4,9 y $1 \mathrm{~m}$, estrato entre 0,9 y $0,5 \mathrm{~m}$ y estrato inferior $<0,5 \mathrm{~m}$ ) y la cobertura global.

Junto a estos datos también se ha recogido otro tipo de información para la valoración complementaria de las comunidades forestales como la cobertura global y la riqueza por estratos (COBEST y RIQUEST), la diversidad de hábitats y sinusias no desglosables a la escala de trabajo (RIQHAB), la superficie de la mancha homogénea (CONESP), la variedaddasonómica tipológica (FISEST, que siempre es $O$ en las comunidades no forestales) y los valores patrimoniales, culturales y etnográficos añadidos (CULEST).

La propuesta metodológica se basa en dos líneas valorativas diferenciadas que suponen, conexiones estrechamente ligadas del sistema operativo (Figura 2).

-El interés de conservación, se calcula siguiendo criterios naturales y culturales y se tiene en cuenta su efectividad diagnóstica y su aplicabilidad real a las escalas de trabajo más habituales; es por ello que, pese a al interés valorativo, no se han tenido en cuenta criterios que precisan gran nivel de detalle. Los criterios de orden natural se sustentan en parámetros fitocenóticos, territoriales, mesológicos y estructurales que aportan información de las propiedades intrínsecas de la flora y de la vegetación, de su corología y de su conexión con el ecosistema en el que se encuetra (Gómez et al., 2014). Las cuestiones de carácter cultural pretenden cuantificar el valor etnobotánico, percepcional, didáctico patrimonial-estructural de la vegetación (Tabla 1). 


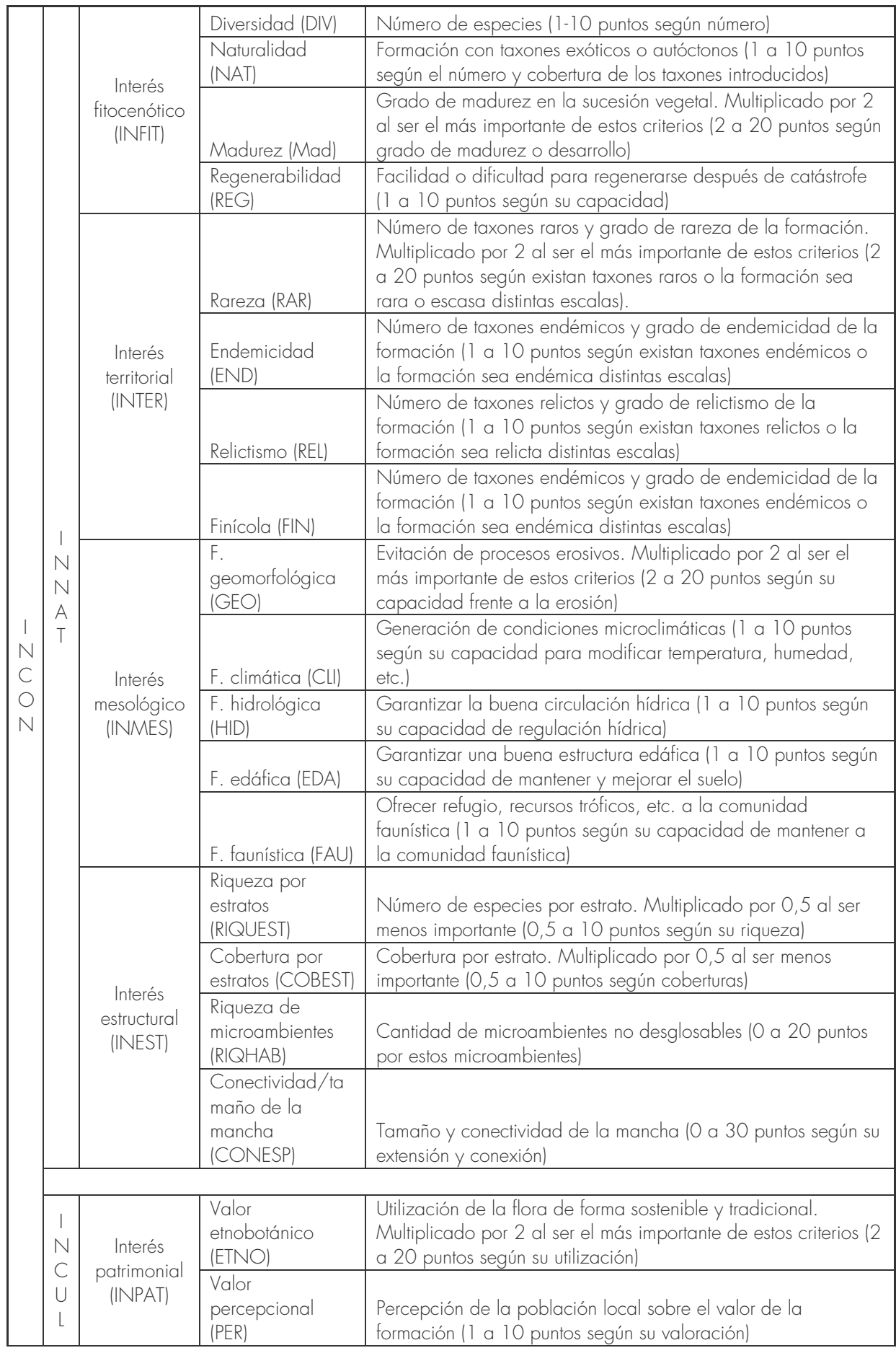

Tabla 1. Criterios del Interés de Conservación (INCON) (elaboración propia)

La prioridad de conservación, continuación evaluar el factor de amenaza ya que tiene del anterior parámetro en la definición y una incidencia directa en cualidades como jerarquización de espacios protegidos está la madurez, rareza, función mesológica o dirigido, en gran medida, hacia la valor etnobotánico desde una perspectiva regulación de la perentoriedad de las dinámica o diacrónica. Al rededor del actuaciones conservacionistas. Es importante concepto de rareza se han elaborado 
concepto de rareza se han elaborado "amenaza", "riesgo" o "peligro" son de uso numerosas metodologías y como resultado habitual al hablar de protección elementos de estos trabajos palabras como naturales y culturales (Tabla 2).

\begin{tabular}{|c|c|c|c|c|}
\hline \multirow{3}{*}{$\begin{array}{l}\text { PR } \\
\text { IC } \\
O \\
N\end{array}$} & \multirow{3}{*}{$\begin{array}{l}A \\
M\end{array}$} & \multirow{3}{*}{$\begin{array}{l}\text { Factor de } \\
\text { Amenaza }\end{array}$} & $\begin{array}{l}\text { Coeficiente de presión } \\
\text { demográfica (DEM) }\end{array}$ & $\begin{array}{l}\text { Densidad de población humana en el territorio ( } 1 \text { a } 10 \text { puntos según } \\
\text { densidad) }\end{array}$ \\
\hline & & & $\begin{array}{l}\text { Coeficiente de } \\
\text { accesibilidad/transitabilidad } \\
\text { (ACT) }\end{array}$ & $\begin{array}{l}\text { Matriz que combina } 6 \text { categorías de accesibilidad y de transitabilidad } \\
\text { (1 a } 10 \text { puntos según esa relación). }\end{array}$ \\
\hline & & & $\begin{array}{l}\text { Coeficiente de amenaza } \\
\text { alternativa (ALT) }\end{array}$ & $\begin{array}{l}\text { Posibilidad de la existencia de otras amenazas naturales o antrópicas } \\
\text { (1 a } 10 \text { puntos según posibilidad) }\end{array}$ \\
\hline
\end{tabular}

Tabla 2. Criterios de la Prioridad de Conservación (INCON) (elaboración propia)

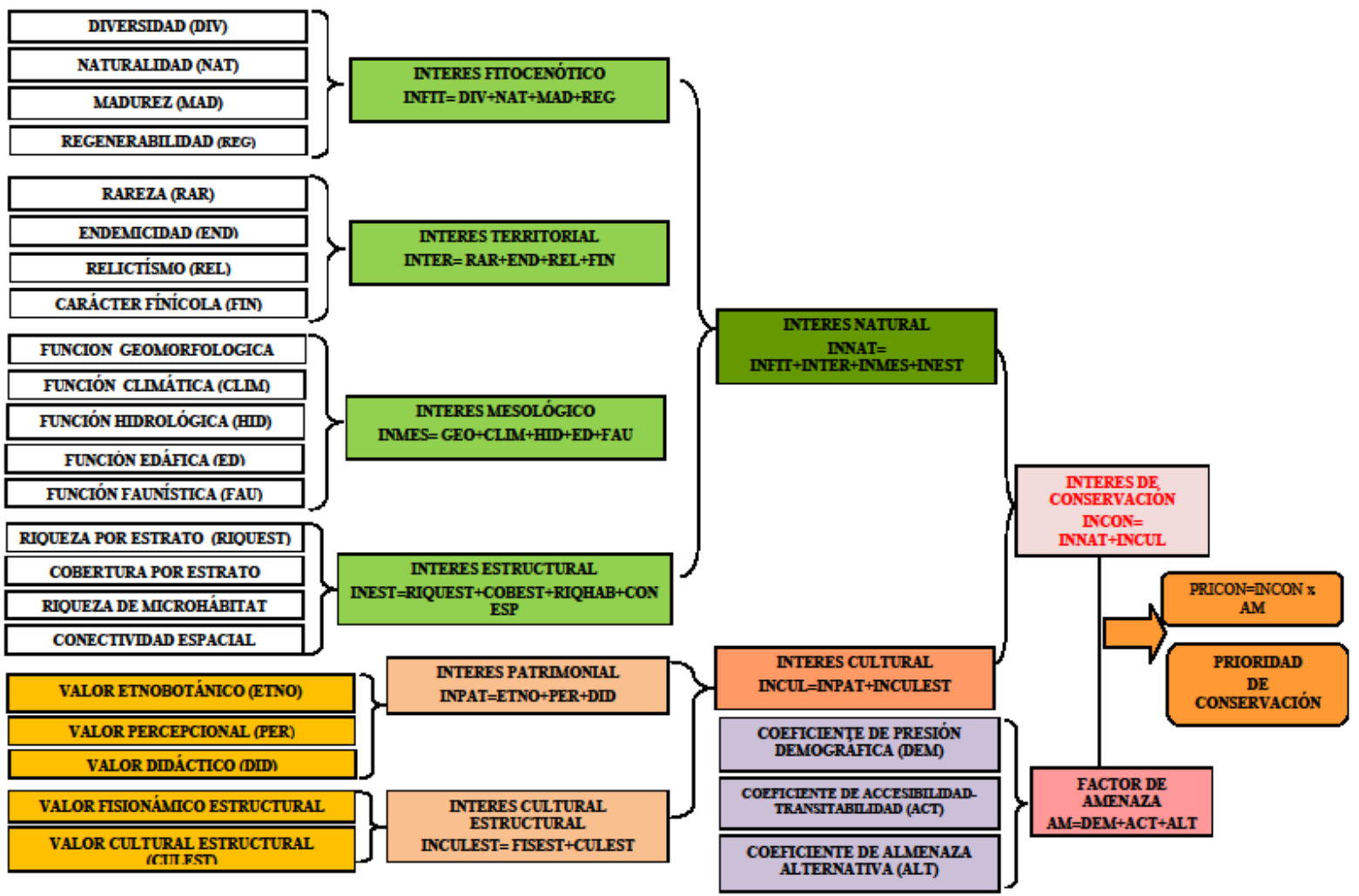

Figura 2. Esquema del método de valoración biogeográfica (elaboración propia). 


\section{RESULTADOS Y DISCUSIÓN}

Los resultados valorativos se plasmaron en Por el contrario, aquellas en las que se 22 inventarios biogeográficos inéditos podían diferenciar distintas facies se (Tabla 3), a la vez que se valoraron y tendió a realizar, al menos, un inventario tomaron los datos oportunos para la por cada una de las variaciones, de estimación de determinados ítems de cara a manera que al final se estiman los la valoración biogeográfica. Estos 22 promedios de los distintos valores de cara inventarios recogen 12 comunidades a facilitar la comprensión de los diferentes (Luebert y Pliscoff, 2006), muchas resultados y aportar un valor único a de ellas mostraban una facies perfectamente formaciones que aunque, con uniforme, de manera que sólo estarán variaciones, cuentan con un cortejo, en representadas a través de un solo inventario. general, relativamente uniforme.

\begin{tabular}{|c|c|c|c|c|c|c|c|}
\hline Lugar & Código & Latitud & Longitud & Altitud & Exposición & Pendiente & Formación \\
\hline 1. Res. Seno Otwai & OTI & $52^{\circ} 59^{\prime} 16^{\prime \prime} \mathrm{S}$ & $\begin{array}{c}71^{\circ} 14^{\prime} 12^{\prime \prime} \\
W\end{array}$ & $3 \mathrm{~m}$ & Todas & $0^{\circ}$ & Tundra herbácea baja \\
\hline 2. Res. Seno Otwai & OT2 & $52^{\circ} 59^{\prime} 18^{\prime \prime} \mathrm{S}$ & $\begin{array}{c}71^{\circ} 14^{\prime} 16^{\prime \prime} \\
W\end{array}$ & $12 \mathrm{~m}$ & Todas & $0^{\circ}$ & Tundra alta-coironal \\
\hline 3. Res. Seno Otwai & ОТ3 & $52^{\circ} 59^{\prime} 18^{\prime \prime} \mathrm{S}$ & $\begin{array}{c}71^{\circ} 15^{\prime} 14^{\prime \prime} \\
W \\
\end{array}$ & $42 \mathrm{~m}$ & Todas & $0^{\circ}$ & Matorral de mata verde \\
\hline 4. Res. Forestal Magallanes & MAGI & $53^{\circ} 08^{\prime} 16^{\prime \prime} \mathrm{S}$ & $\begin{array}{c}71^{\circ} 03^{\prime} 25^{\prime \prime} \\
W\end{array}$ & $465 \mathrm{~m}$ & Sureste & $3-5^{\circ}$ & Lengar abierto \\
\hline 5. Res. Forestal Magallanes & MAG2 & $53^{\circ} 08^{\prime} 48^{\prime \prime} \mathrm{S}$ & $\begin{array}{c}71^{\circ} 01^{\prime} 27^{\prime \prime} \\
W\end{array}$ & $472 \mathrm{~m}$ & Todas & $0-2^{\circ}$ & Lengar denso \\
\hline 6. Res. Forestal Laguna Parrillar & PARI & $53^{\circ} 24^{\prime} 14^{\prime \prime} \mathrm{S}$ & $\begin{array}{c}71^{\circ} 16^{\prime} 30^{\prime \prime} \\
W\end{array}$ & $272 \mathrm{~m}$ & Todas & $0-2^{\circ}$ & Lengar maduro \\
\hline 7. Res. Forestal Laguna Parrillar & PAR2 & $53^{\circ} 24^{\prime} 08^{\prime \prime} \mathrm{S}$ & $\begin{array}{c}71^{\circ} 16^{\prime} 18^{\prime \prime} \\
W\end{array}$ & $280 \mathrm{~m}$ & Sur & $1-2^{\circ}$ & Lengar maduro \\
\hline 8. Res. Forestal Laguna Parrillar & PAR3 & $53^{\circ} 23^{\prime} 57^{\prime \prime} \mathrm{S}$ & $\begin{array}{c}71^{\circ} 15^{\prime} 53^{\prime \prime} \\
W\end{array}$ & $278 \mathrm{~m}$ & Sur & $10-15^{\circ}$ & Lengar en zona fontinal \\
\hline 9. Fuerte Bulnes & BULI & $53^{\circ} 41^{\prime} 02^{\prime \prime} \mathrm{S}$ & $\begin{array}{c}70^{\circ} 58^{\prime} 36^{\prime \prime} \\
W\end{array}$ & $7 \mathrm{~m}$ & Todas & $0^{\circ}$ & Cohigual de Magallanes con lenga \\
\hline 10. Fuerte Bulnes & BUL2 & $53^{\circ} 41^{\prime} 02^{\prime \prime} \mathrm{S}$ & $\begin{array}{c}70^{\circ} 58^{\prime} 36^{\prime \prime} \\
W\end{array}$ & $11 \mathrm{~m}$ & Todas & $1-2^{\circ}$ & Cohigual de Magallanes \\
\hline 11. Torres del Paine & TP1 & $50^{\circ} 56^{\prime} 35^{\prime \prime} \mathrm{s}$ & $\begin{array}{c}72^{\circ} 56^{\prime} 44^{\prime \prime} \\
W\end{array}$ & $752 \mathrm{~m}$ & Noroeste & $7-8^{\circ}$ & Bosque de ñirre \\
\hline 12. Torres del Paine & TP2 & $50^{\circ} 56^{\prime} 37^{\prime \prime} \mathrm{S}$ & $\begin{array}{c}72^{\circ} 56^{\prime} 22^{\prime \prime} \\
W\end{array}$ & $712 \mathrm{~m}$ & Sureste & $6^{\circ}$ & Bosque de ñirre con laderas rezumantes \\
\hline 13. Torres del Paine & TP3 & $50^{\circ} 56^{\prime} 45^{\prime \prime} \mathrm{S}$ & $\begin{array}{c}72^{\circ} 56^{\prime} 08^{\prime \prime} \\
W\end{array}$ & $601 \mathrm{~m}$ & Norte & $32^{\circ}$ & Bosque joven de ñirre \\
\hline 14. Torres del Paine & TP4 & $50^{\circ} 57^{\prime} 56^{\prime \prime} \mathrm{s}$ & $\begin{array}{c}72^{\circ} 53^{\prime} 58^{\prime \prime} \\
\mathrm{W}\end{array}$ & $429 \mathrm{~m}$ & Suroeste & $13^{\circ}$ & Matorral de Notro con ñirre disperso \\
\hline 15. Torres del Paine & TP5 & $50^{\circ} 57^{\prime} 57^{\prime \prime} \mathrm{S}$ & $\begin{array}{c}72^{\circ} 53^{\prime} 53^{\prime \prime} \\
W\end{array}$ & $396 \mathrm{~m}$ & Noroeste & $6^{\circ}$ & Matorral de notro \\
\hline 16. Glaciar Grey & GRE 1 & $51^{\circ} 07^{\prime} 11^{\prime \prime S}$ & $\begin{array}{c}73^{\circ} 07^{\prime} 56^{\prime \prime} \\
W\end{array}$ & $90 \mathrm{~m}$ & Este & $5-25^{\circ}$ & Bosque de ñirre \\
\hline 17. Cuernos del Paine & CUE 1 & $51^{\circ} 00^{\prime} 18^{\prime \prime} \mathrm{S}$ & $\begin{array}{c}73^{\circ} 02^{\prime} 37^{\prime \prime} \\
W\end{array}$ & $317 \mathrm{~m}$ & Norte & $15^{\circ}$ & Cohigual de Magallanes \\
\hline 18. Cuernos del Paine & CUE2 & $51^{\circ} 02^{\prime} 12^{\prime \prime} \mathrm{S}$ & $\begin{array}{c}73^{\circ} 03^{\prime} 12^{\prime \prime} \\
W\end{array}$ & $194 \mathrm{~m}$ & Sur & $4^{\circ}$ & Cohigual de Magallanes \\
\hline 19. Torres del Paine & TP6 & $51^{\circ} 09^{\prime} 54^{\prime \prime} \mathrm{S}$ & $\begin{array}{c}73^{\circ} 03^{\prime} 41^{\prime \prime} \\
W\end{array}$ & $507 \mathrm{~m}$ & Todas & $2^{\circ}$ & Matorral de mata barrosa \\
\hline 20. Torres del Paine & TP7 & $51^{\circ} 09^{\prime} 37^{\prime \prime} \mathrm{S}$ & $\begin{array}{c}73^{\circ} 04^{\prime} 22^{\prime \prime} \\
W\end{array}$ & $554 \mathrm{~m}$ & Todas & $2^{\circ}$ & Matorral de mata guanaco \\
\hline 21. Res. Forestal Magallanes & $S M I$ & $53^{\circ} 09^{\prime} 07^{\prime \prime} \mathrm{S}$ & $\begin{array}{c}71^{\circ} 00^{\prime} 04^{\prime \prime} \\
W\end{array}$ & $311 \mathrm{~m}$ & Todas & $0^{\circ}$ & Parabrezal austral \\
\hline 22. Res. Forestal Magallanes & SM2 & $53^{\circ} 09^{\prime} 38^{\prime \prime} \mathrm{S}$ & $\begin{array}{c}71^{\circ} 00^{\prime} 52^{\prime \prime} \\
W\end{array}$ & $327 \mathrm{~m}$ & Todas & $0^{\circ}$ & Turbera \\
\hline
\end{tabular}

Tabla 3. Características Geográficas de los inventarios (elaboración propia). Res.: Reserva.

Los resultados de cada uno de los inventarios aparecen recogidos en la tabla 4 . Aunque, como queda dicho, sobre el terreno se tomaron los datos de cobertura de cada taxón para los cuatro estratos de altura diferentes, en la tabla sólo se recoge el dato global de la cobertura. El número total de especies vasculares es de 97, repartidas de la siguiente manera: 5 árboles y arbustos altos, 22 matas y trepadoras y 70 herbáceas. 


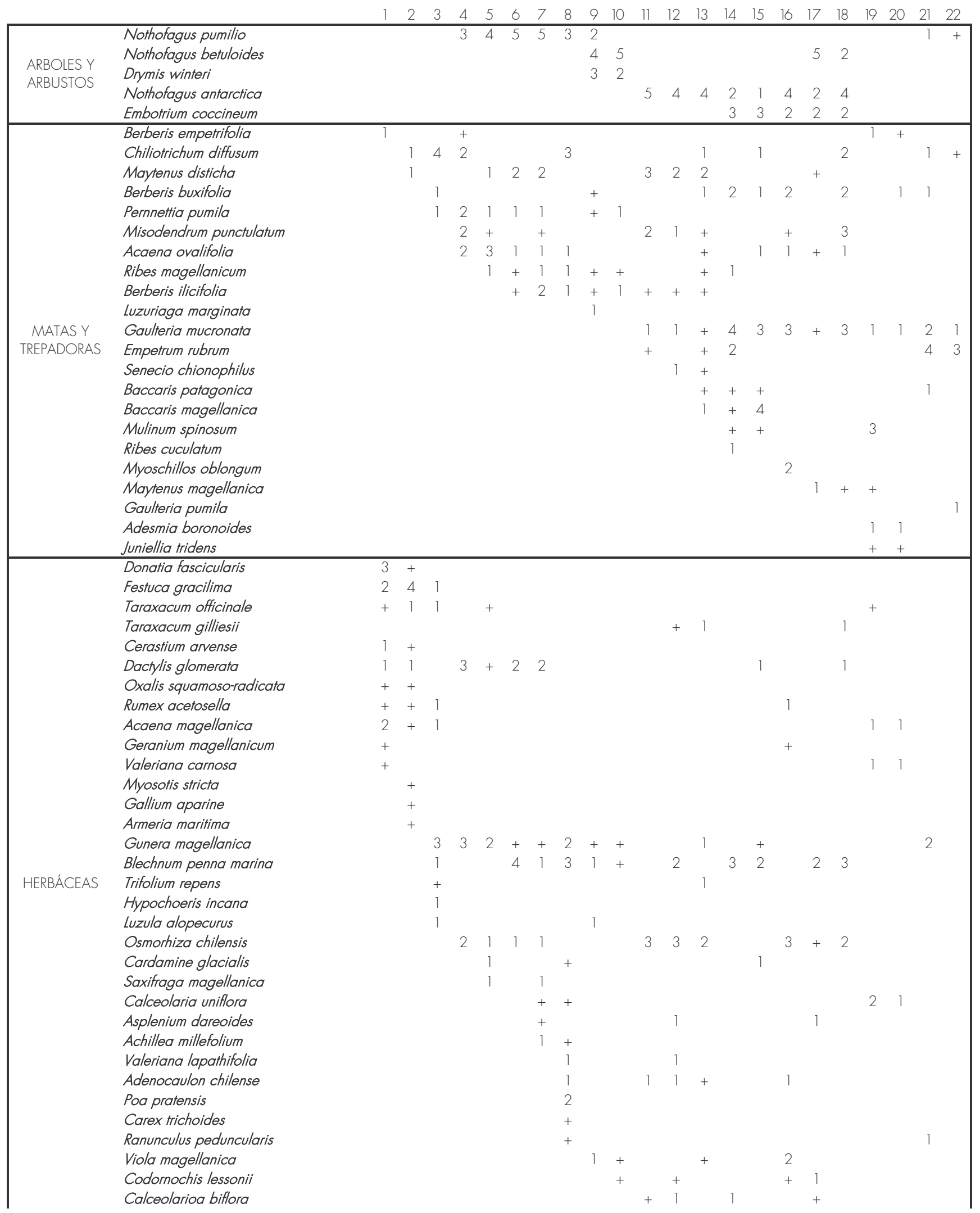


Bolax gumifera Acaena pinatifida

Lathyrus nervosus

Hypochoeris radicata

Gavilea kingii

Plantago lanceolata

Anemone multifida

Luzula magellanica

Gavilea lutea

Deschampsia flexuosa

Holcus lanatus

Poa pratensis

Poa patagonica

Senecio chilensis

Senecio tricuspidatus

Astenia pumila

Festuca magellanica

Carex magellanica

Marsippospermum grandiflorum

Draba magellanica

Primula magellanica

Chloraea magellanica

Cerastium arvense

Hordeum comosum

Viola maculata

Olsinium biflorum

Senecio patagonicus

Elymus angulatus

Adesmia salicornioides

Phacelia secunda

Calceolaria tenella

Líquenes en troncos y ramas

Líquenes en rocas y suelo

Musgos en troncos y ramas

Musgos en rocas y suelo

Hongos

Hojarasca

Suelo o roca desnuda

Tabla 4. Inventarios biogeográficos y coberturas globales por especie (elaboración propia). 


\section{CARACTERIZACIÓN Y DESCRIPCIÓN DE LAS DISTINTAS FORMACIONES}

\section{TUNDRA}

En la zona austral de Chile, aunque bastante abundante en latitudes más meridionales, las comunidades de tundra se rarifican a medida que se avanza hacia el norte (Guerrido y Fernández, 2007). Es en la Península Brunswick, más concretamente en el sector de Seno Otway, donde se pudieron inventariar dos ejemplos con facies diferentes de tundra. El primero se corresponde con una tundra baja, mientras el segundo lo hace con una tundra un poco más alta, tanto porque se sitúa unos metros de altura por encima de la primera, como por la irrupción de especies de mayor porte, aunque estamos hablando de una comunidad representada en todo caso por especies de pequeño porte y achaparradas para resistir las duras condiciones bioclimáticas de esta zona (Figura 3). En el primer caso la capa freática se encuentra muy próxima a la superficie $y$, de hecho, en las pequeñas depresiones existentes aparece en superficie. En el segundo, puede estar a unos dos o más metros de profundidad. En cualquier caso la fisonomía de esta tundra es la de un tapiz muy compacto con una gran densidad de herbáceas o arbustos de escasa talla. Son propias de estas comunidades especies como: Berberis empetrifolia, Donatia fascicularis, Acaena magellanica, Oxalis squamoso-radicata. También son visibles plantas propias del coironal y, por tanto, con mayor porte, como Festuca gracillima. Hay que destacar también la gran cantidad de especies introducidas: Dactylis glomerata, Taraxacum officinale o Rumex acetosella.

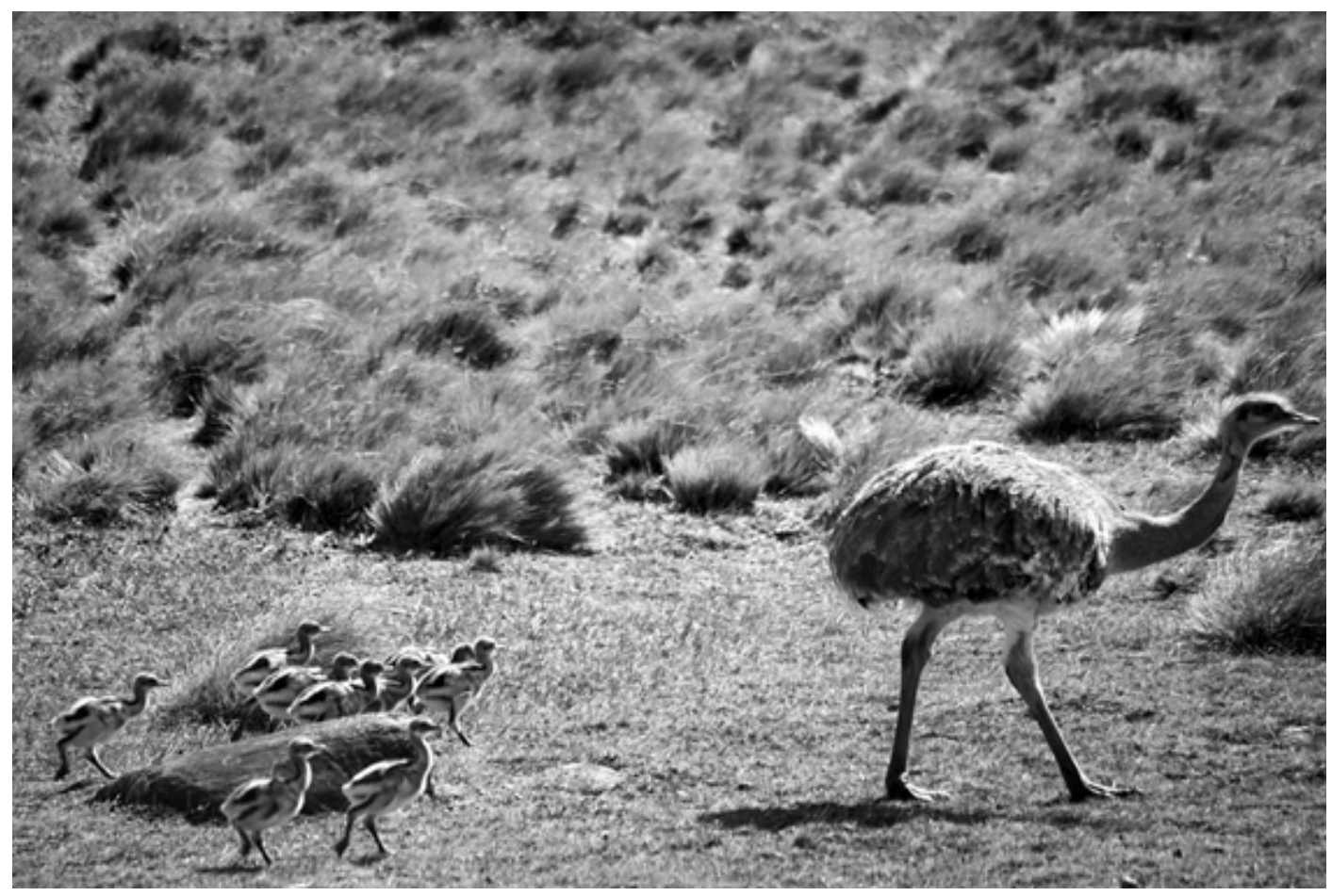

Figura 3. Tundra herbosa en Seno Otway (fotografía de los autores). 
PARABREZAL AUSTRAL

Se trata de etapas degradadas 0 de sustitución de otras comunidades, fundamentalmente asociadas al bosque de lengas (Nothofagus pumilio), de la cual es estadío previo en la sucesión. Aparece en los bordes del bosque y ostenta una fisonomía baja, de menos de medio metro de altura, pero, a la vez, bastante densa y compacta. Normalmente soporta bien los suelos de naturaleza gleyca, bastante húmedos, y, por ello, incluso suele aparecer al lado de turberas, como en el caso del inventario realizado. La dinámica natural tiende a hacer derivar estas comunidades hacia prebosques $\mathrm{O}$ matorrales más altos e intrincados entre los que van apareciendo ejemplares de lenga, coigüe de Magallanes (Nothofagus betuloides) 0 incluso ñirre (Nothofagus antarctica). El cortejo de vegetación propio incluye especies arbustivas de bajo porte como: Empetrum rubrum, taxón más abundante, Gaulteria mucronata, Gaulteria pumila y herbáceas como: Marsippospermum grandiflorum, Carex magellanica, Festuca magellanica, Luzula magellanica, Astenia pumila, Deschampsia flexuosa, Lycopodium magellanicum y otros.

\section{TURBERA AUSTRAL}

Ocupa grandes extensiones de gleysoles, permanentemente encharcados o incluso con permafrost. En el inventario realizado se caracterizó una turbera contigua a la mancha de parabrezal austral, aunque ocupando, lógicamente, los sectores más deprimidos y con mal drenaje. Su fisonomía es la de un tapiz herbáceo disperso o en macollas entre canales y pequeñas depresiones encharcadas. No obstante, las islas o sectores donde aparece la vegetación higrófila muestra un tapiz bajo, de gran densidad y muy compacto. En este caso y, en general, estas turberas muestran un cortejo de especies pobre y muy reducido en taxones. Suelen ser típicas especies como: Marsippospermum grandiflorum, Empetrum rubrum, Gaulteria pumila y Gaulteria mucronata así como alguna herbáceas: Carex magellanica, Deschampsia flexuosa, Carex canenscens, etc. (Quintanilla, 1989).

\section{MATORRAL DE MATA VERDE (CHILIOTRICHUM DIFFUSUM)}

Se trata de una asociación arbustiva que se establece entre las áreas de bosque, fundamentalmente lenga, y las zonas o sectores de estepa patagónica. Aunque pueden presentar una fisonomía más abierta, normalmente se corresponde a un matorral de talla media, entre 0,5 y $1 \mathrm{~m}$, con una estructura intrincada, cerrada y muchas veces compacta. Junto a Chiliotrichum diffusum, que muestra unas coberturas entre el 50 y $75 \%$, se pueden identificar otras especies, normalmente pequeños arbustos o herbáceas. Entre los primeros habría que destacar: Berberis buxifolia y Pernettia pumila, $y$, entre las segundas: Festuca gracilima, Acaena magellanica, Gunera Magellanica, Blechnum penna marina, Trifolium repens, Hypochoeris incana, Luzula alopecurus... así como especies introducidas: Oxalis acetosella y Taraxacum officinale.

\section{MATORRAL DE NOTRO (EMBOTRIUM COCCINEUM)}

Etapa de sustitución del bosque de ñirre. Se establece en sectores de una altitud elevada, entre 1.000 y 1.500 metros, y suele responder a zonas donde se han dado procesos naturales 0 antrópicos de deforestación. Se trata de una etapa previa a la aparición de un bosque joven $y$, de hecho, en el presente artículo quedan 
reflejadas dos facies, una más arbustiva, mientras que la otra mostraba evidentes y abundantes pies jóvenes de ñirre. De esta forma, la fisonomía característica es la de un matorral más o menos abierto, con abundancia de pies de entre uno y tres metros de altura y con un tapiz bajo y apretado de Empetrum rubrum, Gaulteria mucronata, Ribes magellanicum, Berberis buxifolia, Baccaris patagonica, Baccaris magellanica y herbáceas: Blechnum penna-marina, Festuca pallescens, Lycopodium magellanicum, Bolax gumifera y Acaena pinatifida.

En los dos inventarios realizados, Embotrium coccineum mostraba una cobertura superior al $50 \%$, pero ya aparecían pies más o menos aislados de ñirre con densidades entre el 10 y el $25 \%$.

\section{MATORRAL DE MATA BARROSA (MULINUM SPINOSUM)}

El matorral de mata barrosa ocupa importantes extensiones fuera del bosque, en espacios a una cierta altitud, entre 800 y $1.500 \mathrm{~m}, \mathrm{y}$, en muchos casos, responde a una etapa paraclimácica de laderas con escasos suelos, litosoles normalmente, o con una orientación muy expuesta a los flujos fríos pero a la vez, con un déficit hídrico permanente derivado del carácter rocoso de los suelos, de manera que algunos autores lo denominan "matorral xerófito pre-andino" (Garay y Guineo, 2003: 183). Estas condiciones realmente duras permiten únicamente la pervivencia de un matorral poco tupido, muy ramoneado por el guanaco y que muestra una fisonomía en manchas apretadas y densas de la especie dominante, Mulinum spinosum, con portes entre 50 y 90 cm, junto con Maytenus magellanica, Berberis empetrifolia, Adesmia boronoides, Gaulteria mucronata, Juniella tridens y, en aquellos sectores con suelos un poco más desarrollados, Anarthrophyllum desideratum, todos ellos de porte subarbustivo. En cuanto a herbáceas habría que destacar: Draba magellanica, Primula magellanica, Valeriana carnosa, Chloraea magellanica, Cerastium arvense, Hordeun comosum, Olsinium biflorum, Calceolaria uniflora, etc.

\section{MATORRAL DE MATA GUANACO (ANARTHROPHYLLUM DESIDERATUM)}

Como bien indica su nombre, esta comunidad se encuentra caracterizada por el predominio de Anarthrophyllum desideratum, mata muy apreciada por el guanaco y muy conspicua en su antesis por sus flores de un llamativo rojo. La suma de unas condiciones ambientales duras más el sistemático ramoneo del camélido, muy abundante en algunos sectores, da como resultado que las matas apenas sobrepasen los 40 a $70 \mathrm{~cm}$ de porte. Mantiene, por tanto, una fisonomía muy similar a la mata barrosa y de hecho comparten y se reparten prácticamente los mismos territorios y características ambientales, con la diferencia de que la mata guanaco (Figura 4) precisa suelos más profundos y, por ello, coloniza las vertientes con exposiciones menos expuestas, recuencos, pies de zonas rocosas con mayores y mejores suelos, etc. En cualquier caso, su cortejo florístico es muy similar al de la unidad anterior: Maytenus magellanica, Berberis buxifolia, Berberis empetrifolia, Adesmia boronoides, Gaulteria mucronata y Juniella tridens. En cuanto a herbáceas habría que destacar: Draba magellanica, Primula magellanica, Valeriana carnosa, Acaena magellanica, Adesmia salicornoides, Chloraea magellanica, Cerastium arvense, Hordeun comosum, Olsinium biflorum, Calceolaria uniflora, Calceolaria tenella y Festuca magellanica. 


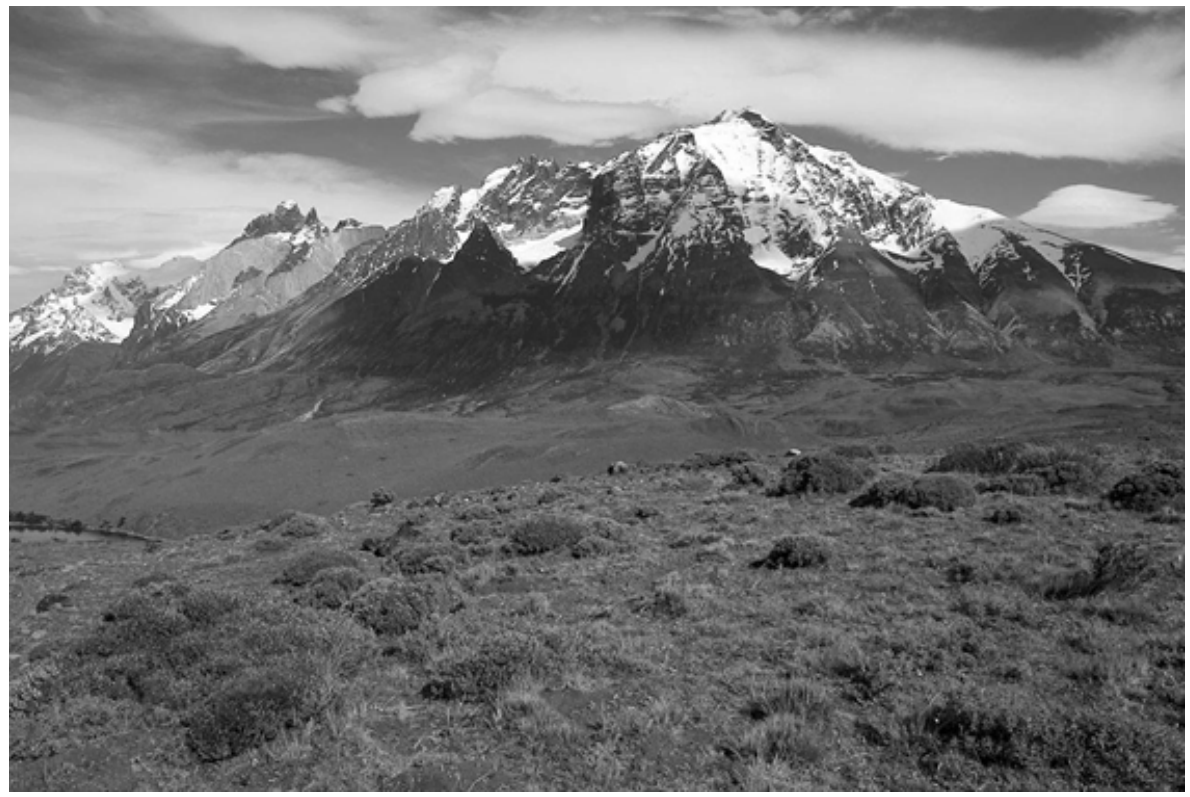

Figura 4. En primer plano, matorral de mata guanaco (fotografía de los autores).

\section{BOSQUE DE LENGA (NOTHOFAGUS PUMILIO)}

Los bosques de lenga (Figura 5) se localizan Se da entonces una transición, de manera principalmente en los archipiélagos que, conforme ascendemos, va dando paso meridionales de Magallanes. En nuestro caso al ñirre (Nothofagus Antarctica). Por su parte, las observaciones fueron realizadas en la el coigüe de Magallanes (Nothofagus Península de Brunswick, donde se configura betuloides) puede ser encontrado a diversas como el bosque dominante a cualquier altitud. altitudes y diferentes sectores, de manera que Fuera de aquí, en sectores más continentales y muchas veces aparece formando bosques cercanos al Parque Nacional de Torres del mixtos con cualquiera de las otras dos Paine,ocupa zonas de baja altitud. especies citadas anteriormente.

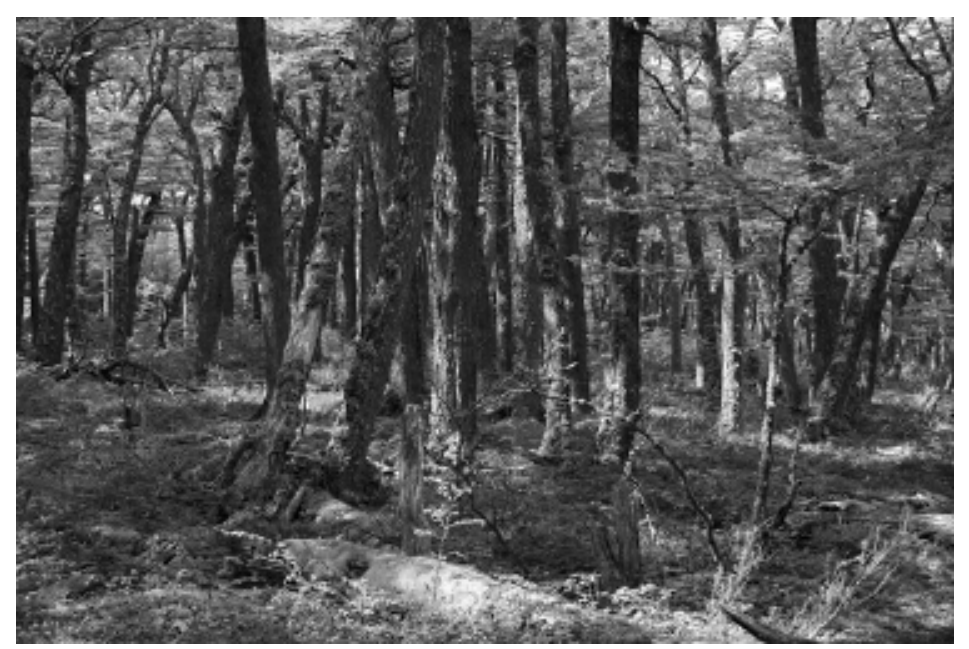

Figura 5. Interior de un bosque de lenga en El Parrillar (fotografía de los autores). 
Los bosques de lenga muestran facies diferenciadas que dependen de cuestiones muy variadas como lo expuesto de los sectores que ocupen a los flujos constantes de vientos fríos y húmedos del suroeste y oeste. También dependerá de la mayor o menor profundidad y desarrollo edáfico y, en mayor medida, de la presión antrópica a la que hayan sido sometidos. Muchos de ellos fueron explotados a través de técnicas bastante selectivas (entresaca), de manera que muestran bosques abiertos con ejemplares muy añosos, barbados (por la gran profusión de líquenes) y arrumbados cuando no totalmente abanderados /por la constante presión e influencia de los vientos dominantes). En otros sectores donde no han sido sometidos a estas presiones muestran fustes más rectilíneos, con mayor altura y con mayor profusión de pies. En los cinco inventarios realizados de esta tipología, el único ocupante del estrato arbóreo o arbustivo era la propia lenga, lo que recuerda al vuelo de los hayedos del Hemisferio Norte. En cualquier caso, cuentan con un cortejo florístico que puede ser diferente si muestran bajas densidades, ya que entonces aparecen plantas de otras unidades, como el matorral de mata verde - la estepa, o, si la densidad es mayor es mucho más pobre. Las especies que más a menudo aparecen asociadas a estos bosques y englobadas dentro de portes subarbustivos o escandentes son: Chiliotrichum diffusum, Maytenus disticha, Pernnettia pumila, Misodendrum punctulatum, Acaena ovalifolia, Ribes magellanicum y Berberis ilicifolia; mientras que las herbáceas más recurrentes son: Dactylis glomerata, Gunera magellanica, Blechnum penna marina, Osmorhiza chilensis, Cardamine glacialis, Saxifraga magellanica, Poa pratensis y Valeriana laphatifolia.

\section{BOSQUE DE COIGÜE DE} MAGALLANES (NOTHOFAGUS BETULOIDES)

Los bosques de coigüe de Magallanes no son tan abundantes como los de lenga y ñirre, pero, al contrario que estos que muestran un reparto según altitudes en el sector estudiado, se reparten por todos los sectores y muchas veces forman bosques mixtos, como se describirá posteriormente. Son, en cualquier caso, bosques pobres florística y estructuralmente y suelen ocupar suelos relativamente bien drenados pero, a la vez, en sectores con abundantes precipitaciones que suelen rondar los 3000 mm/año (Pisano, 1977). Tienden a ser bosques con fustes muy abundantes y rectilíneos, como consecuencia de la gran competencia que se establece por la luz. El que mantengan la hoja durante todo el año y que, además, muestren esta gran densidad de fustes y muchas veces follaje, hace que la luz solar que pueda llegar al suelo sea muy escasa $y$, por lo tanto, exista una crónica penuria de especies en el sotobosque $y$, aquellas que aparecen, muestren, en todo caso, unas características umbrófilas evidentes.

Junto al coigüe suele aparecer otra especies arbórea como es el canelo (Drymis winteri). Las matas y trepadoras son escasas pudiéndose apuntar fundamentalmente Luzuriaga marginata. En cuanto a las escasísimas herbáceas, reseñar: Blechnum penna-marina, Luzula alopecurus, Asplenium dareoides, Viola magellanica y Deschampsia flexuosa.

\section{BOSQUES DE ÑIRRE (NOTHOFAGUS ANTARCTICA)}

Es el tercer gran tipo de bosque de estos sectores de la Patagonia chilena. La especie dominante es el ñirre que puede venir acompañado por otros árboles como el 
coigüe de Magallanes (Nothofagus betuloides) o incluso especies arbustivas como el notro (Embotrium coccineum). Sin embargo, es ciertamente difícil detectar bosques mixtos con lenga (Nothofagus pumilio). De hecho, como se apuntaba en el epígrafe 8, existe una división territorial, de manera que el ñirre ocupa los espacios de mayor altitud $y$, por tanto, las zonas cordilleranas con los necesarios espesores de suelo. No obstante, en aquellos sectores de la Cordillera que sobrepasan los 800 metros y cuentan con suelos no demasiado profundos y, a la vez, muy azotados por los flujos de vientos más o menos constantes, el bosque aparece achaparrado, con alturas arbustivas o subarbóreas que nunca sobrepasan los $5 \mathrm{~m}$.

Los bosques de ñirre también han sufrido en estas latitudes y sectores una gran presión antrópica de manera que, en muchos casos, incluso dentro de estos espacios protegidos, la mayor parte de ellos muestran facies de bosque joven puesto que sufrieron una explotación forestal indiscriminada. Son escasos los ejemplos de bosques maduros. En nuestro caso detectamos uno en la ribera sur del lago Grey que mostraba signos de haber sufrido una explotación selectiva por entresaca. El cortejo consta de matas como: Chiliotrichum diffusum, Maytenus disticha, Berberis ilicifolia, Gaulteria mucronata, Senecio chionophilus, la parásita Misodendrum punctulatum, y herbáceas como: Osmorhiza chilensis, Adenocaulon chilense, Vicia magellanica, Codornochis lessonii, Viola magellanica, Anemone multifida, Gavilea lutea, Holcus lanatus, Deschampsia flexuosa, etc.

estudiado, se reparten por todos los sectores y muchas veces forman bosques mixtos, como se describirá posteriormente. Son, en cualquier caso, bosques pobres florística y estructuralmente y suelen ocupar suelos relativamente bien drenados pero, a la vez, en sectores con abundantes precipitaciones que suelen rondar los 3000 mm/año (Pisano, 1977). Tienden a ser bosques con fustes muy abundantes y rectilíneos, como consecuencia de la gran competencia que se establece por la luz. El que mantengan la hoja durante todo el año y que, además, muestren esta gran densidad de fustes y muchas veces follaje, hace que la luz solar que pueda llegar al suelo sea muy escasa y, por lo tanto, exista una crónica penuria de especies en el sotobosque $y$, aquellas que aparecen, muestren, en todo caso, unas características umbrófilas evidentes.

Junto al coigüe suele aparecer otra especies arbórea como es el canelo (Drymis winteri). Las matas y trepadoras son escasas pudiéndose apuntar fundamentalmente Luzuriaga marginata. En cuanto a las escasísimas herbáceas, reseñar: Blechnum penna-marina, Luzula alopecurus, Asplenium dareoides, Viola magellanica y Deschampsia flexuosa.

\section{BOSQUES MIXTOS DE LENGA O ÑIRRE CON COIGÜE DE MAGALLANES}

Como se ha comentado anteriormente, son bastante abundantes los ejemplos de bosques mixtos donde el coigüe de Magallanes aparece asociado a bosquetes de lenga o de ñirre. No hemos podido comprobar, no obstante, un bosque mixto entre las últimas dos especies citadas. En cualquier caso, estos bosques adquieren diferentes facies $y$, a la vez, reúnen las especies acompañantes de los dos tipos de bosque que se mezclan. De esta forma, pueden aparecer especies arbustivas como Drymis winteri y Embotrium coccineum, matas como Berberis buxifolia, Pernettia pumilia, 
Maytenus disticha, Maytenus magellanica, VALORACIÓN BIOGEOGRÁFICA DE Acaena ovalifolia o Luzuriaga marginata, LOS PAISAJES VEGETALES DESCRITOS y herbáceas como Blechnum penna-marina, Luzula alopecurus, Asplenium dareoi- A continuación se aborda el análisis des, Viola magellanica, Codornochis detallado de las puntuaciones obtenidas lessonii, Calceolaria biflora, Deschampsia según los distintos criterios y valores por cada flexuosa, Senecio chilensis y Senecio tricus- uno de los paisajes vegetales inventariados y pidatus. valorados (Tabla 5).

\begin{tabular}{|c|c|c|c|c|c|c|c|c|c|c|c|c|c|}
\hline \multirow[b]{2}{*}{ COMUNIDADES } & \multirow[b]{2}{*}{ VARIANTES O MATIZACIONES } & \multirow{2}{*}{$\begin{array}{l}\text { CODIGO } \\
\text { INV. }\end{array}$} & \multicolumn{11}{|c|}{ valoración } \\
\hline & & & $\begin{array}{c}\text { Infi } \\
t\end{array}$ & Inter & $\begin{array}{c}\text { Inme } \\
s\end{array}$ & Inest & Innat & $\begin{array}{c}\text { Inp } \\
\text { at }\end{array}$ & $\begin{array}{c}\text { Incules } \\
t\end{array}$ & $\begin{array}{c}\text { Inc } \\
\text { ul }\end{array}$ & Incon & $\begin{array}{l}\mathrm{A} \\
\mathrm{M}\end{array}$ & Pricon \\
\hline TUNDRA & Tundra baja herbácea & OT1 & 42 & 15 & 37 & $\begin{array}{c}78 \\
5\end{array}$ & $\begin{array}{c}172 \\
5\end{array}$ & 31 & 0 & 31 & 203,5 & 8 & 1628 \\
\hline TUNDRA & Tundra alta y coironal & OT2 & 39 & $\begin{array}{c}16 \\
3\end{array}$ & 38 & $\begin{array}{c}77 \\
5\end{array}$ & $\begin{array}{c}170 \\
8\end{array}$ & 22 & 0 & 22 & 192,8 & 8 & 1542,4 \\
\hline \multicolumn{3}{|c|}{ VALORACIÓN MEDIA DE LA TUNDRA } & $\begin{array}{r}40 \\
5\end{array}$ & $\begin{array}{l}15 \\
65\end{array}$ & 37,5 & 78 & $\begin{array}{c}171 \\
65\end{array}$ & $\begin{array}{c}26, \\
5\end{array}$ & 0 & $\begin{array}{c}26, \\
5\end{array}$ & $\begin{array}{c}198,1 \\
5\end{array}$ & 8 & 1585,2 \\
\hline PARABREZAL AUSTRAL & Parabrezal austral & SMI & 27 & 9,1 & 40 & $\begin{array}{c}16 \\
5\end{array}$ & 92,6 & 12 & 0 & 12 & 104.6 & 16 & 1673,6 \\
\hline TURBERA AUSTRAL & Turbera austral & SM2 & 37 & 4 & 34 & $\begin{array}{c}12 \\
5\end{array}$ & 87,5 & 16 & 0 & 16 & 103,5 & 14 & 1449 \\
\hline MATORRAL DE MATA VERDE & Matorral de mata verde & ОТ3 & 29 & 3,8 & 38 & 79 & $\begin{array}{c}149, \\
8\end{array}$ & 20 & 4 & 24 & 173,8 & 8 & 1390,4 \\
\hline MATORRAL DE NOTRO & $\begin{array}{l}\text { Matorral de notro con ñirre } \\
\text { disperso }\end{array}$ & TP4 & 32 & $\begin{array}{c}15 \\
5 \\
\end{array}$ & 40 & $\begin{array}{c}28, \\
5\end{array}$ & 116 & 12 & 2 & 14 & 130 & 14 & 1820 \\
\hline MATORRAL DE NOTRO & Matorral de notro & TP5 & 30 & $\begin{array}{c}18 \\
5\end{array}$ & 40 & 28 & $\begin{array}{c}116 \\
5\end{array}$ & 18 & 2 & 20 & 136,5 & 14 & 1911 \\
\hline \multicolumn{3}{|c|}{ VALORACIÓN MEDIA DEL MATORRAL DE NOTRO } & 31 & 17 & 40 & $\begin{array}{l}28 \\
25\end{array}$ & $\begin{array}{l}116 \\
25\end{array}$ & 15 & 2 & 17 & $\begin{array}{c}133,2 \\
5\end{array}$ & 14 & 1865,5 \\
\hline MATORRAL DE MATA BARROSA & Matorral de mata barrosa & TP6 & 32 & $\begin{array}{c}16 \\
5\end{array}$ & 40 & 22 & $\begin{array}{c}110 \\
5\end{array}$ & 21 & 2 & 23 & 133,5 & 14 & 1869 \\
\hline MATORRAL DE MATA GUANACO & Matorral de mata guanaco & TP7 & 32 & 27 & 41 & 17 & 117 & 27 & 2 & 29 & 146 & 14 & 2044 \\
\hline LENGAR & Lengar abierto y maduro & MAGI & 33 & 4,9 & 51 & $\begin{array}{c}57 \\
5\end{array}$ & $\begin{array}{c}146 \\
4\end{array}$ & 28 & 8 & 36 & 182,4 & 8 & 1459,2 \\
\hline LENGAR & Lengar denso & MAG2 & 37 & $\begin{array}{c}12 \\
6\end{array}$ & 49 & 57 & $\begin{array}{c}155 \\
6\end{array}$ & 26 & 2 & 28 & 183,6 & 11 & 2019,6 \\
\hline LENGAR & Lengar maduro & PAR 1 & 39 & 9 & 54 & $\begin{array}{c}59 \\
5\end{array}$ & $\begin{array}{c}161 \\
5\end{array}$ & 34 & 4 & 38 & 199,5 & 8 & 1596 \\
\hline LENGAR & Lengar maduro & PAR2 & 40 & $\begin{array}{c}11 \\
3\end{array}$ & 54 & $\begin{array}{c}58 \\
5\end{array}$ & $\begin{array}{c}163 \\
8\end{array}$ & 34 & 4 & 38 & 201,8 & 8 & 1614,4 \\
\hline LENGAR & $\begin{array}{l}\text { Lengar maduro en ladera } \\
\text { rezumante }\end{array}$ & PAR3 & 42 & 14 & 54 & 58 & 168 & 25 & 2 & 27 & 195 & 8 & 1560 \\
\hline \multicolumn{3}{|c|}{ VALORACIÓN MEDIA DEL LENGAR } & $\begin{array}{r}38 \\
2\end{array}$ & $\begin{array}{l}10, \\
36\end{array}$ & 52,4 & 58, & $\begin{array}{c}159, \\
06\end{array}$ & $\begin{array}{c}29, \\
4\end{array}$ & 4 & $\begin{array}{c}33, \\
4\end{array}$ & $\begin{array}{c}192,4 \\
6\end{array}$ & 8,6 & $\begin{array}{c}1649,8 \\
4\end{array}$ \\
\hline BOSQUE MIXTO COHIGUAL-LENGAR & Bosque mixto cohigual-lengar & BUL 1 & 34 & $\begin{array}{c}14 \\
6\end{array}$ & 47 & $\begin{array}{c}54 \\
5\end{array}$ & $\begin{array}{c}150 \\
1\end{array}$ & 33 & 4 & 37 & 187,1 & 13 & 2432,3 \\
\hline BOSQUE MIXTO COHIGUAL-ÑIRRE & Bosque mixto cohigual-ñirre & CUE 1 & 41 & $\begin{array}{c}14 \\
2\end{array}$ & 45 & $\begin{array}{c}29, \\
5\end{array}$ & $\begin{array}{c}129 \\
7\end{array}$ & 19 & 2 & 21 & 150,7 & 10 & 1507 \\
\hline COHIGUAL DE MAGALLANES & Cohigual de Magallanes maduro & BUL2 & 36 & 8,4 & 38 & $\begin{array}{c}49 \\
5\end{array}$ & $\begin{array}{c}131 \\
9\end{array}$ & 16 & 4 & 20 & 151,9 & 14 & 2126,6 \\
\hline COHIGUAL DE MAGALLANES & Cohigual de Magallanes joven & CUE2 & 34 & $\begin{array}{c}16 \\
8\end{array}$ & 42 & 22 & $\begin{array}{c}114 \\
8\end{array}$ & 13 & 2 & 15 & 129,8 & 9 & 1168,2 \\
\hline \multicolumn{3}{|c|}{ VALORACIÓN MEDIA DEL COHIGUAL DE MAGALLANES } & 35 & $\begin{array}{c}12 \\
6\end{array}$ & 40 & $\begin{array}{l}35 \\
75\end{array}$ & $\begin{array}{c}123 \\
35\end{array}$ & $\begin{array}{c}14, \\
5\end{array}$ & 3 & $\begin{array}{c}17, \\
5\end{array}$ & $\begin{array}{c}140,8 \\
5\end{array}$ & $\begin{array}{c}11, \\
5\end{array}$ & 1647,4 \\
\hline BOSQUE DE ÑIRRE & Bosque de ñirre maduro & TP1 & 41 & $\begin{array}{c}12 \\
2\end{array}$ & 48 & $\begin{array}{c}38 \\
5\end{array}$ & $\begin{array}{c}139 \\
7\end{array}$ & 34 & 6 & 40 & 179,7 & 9 & 1617,3 \\
\hline BOSQUE DE ÑIRRE & Bosque de ñirre & TP2 & 39 & $\begin{array}{c}17 \\
6\end{array}$ & 49 & $\begin{array}{c}36 \\
5\end{array}$ & $\begin{array}{c}142 \\
1\end{array}$ & 29 & 4 & 33 & 175,1 & 8 & 1400,8 \\
\hline BOSQUE DE ÑIRRE & Bosque de ñirre joven & TP3 & 34 & $\begin{array}{c}21, \\
4\end{array}$ & 53 & 36 & $\begin{array}{c}144 \\
4\end{array}$ & 29 & 2 & 31 & 175,4 & 10 & 1754 \\
\hline BOSQUE DE NIIRRE & Bosque de ñirre maduro y abierto & GRE I & 38 & 30 & 52 & 24 & 144 & 28 & 6 & 34 & 178 & 15 & 2670 \\
\hline
\end{tabular}

Tabla 5. Tabla sinóptica de comunidades inventariadas en la Patagonia Chilena y de su valoración biogeográfica. Elaboración propia. Ver el significado de los acrónimos de los índices en las tablas 1 y 2. 
Como se observa en la Tabla 5, la y PRICON. En cualquier caso, por mor de clasificación valorativa de las diferentes la brevedad nos vamos a centrar en tres comunidades vegetales fluctúa parámetros fundamentales: el INNAT, dependiendo de los aspectos que se donde se reflejan los criterios y aspectos tomen en cuenta. Precisamente esta es una puramente naturales, el INCON, donde a de las grandes virtualidades del método, los valores naturales se suman los de puesto que se puede realizar una lectura naturaleza cultural, y, por último, el parcial, dependiendo de los PRICON, prioridad de conservación que requerimientos del gestor o tomador de estima los valores relacionados con el decisiones en cuanto a priorizar un grado de presión antrópica y los riesgos determinado criterio o, al contrario, tomar susceptibles de afectar a cada comunidad en cuenta valores globales como INCON (Tabla 6).

\begin{tabular}{|l|c|c|c|c|c|c|}
\hline \multirow{2}{*}{ COMUNIDADES } & \multicolumn{3}{|c|}{ valoración } & \multicolumn{3}{c|}{ clasificación } \\
\cline { 2 - 7 } & INNAT & INCON & PRICON & INNAT & INCON & PRICON \\
\hline TUNDRA & 85,15 & 176,65 & 1413 & Nirre & Tundra & Mixto C/L \\
\hline PARABREZAL AUSTRAL & 72,1 & 94,6 & 1513 & Lenga & Lenga & Mata guanaco \\
\hline TURBERA AUSTRAL & 75 & 97,5 & 1365 & Mata guanaco & Mixto C/L & Notro \\
\hline MATORRAL DE MATA VERDE & 63,8 & 154,8 & 1238 & Mixto C/N & Nirre & Nirre \\
\hline MATORRAL DE NOTRO & 86 & 123,25 & 1725 & Mixto C/L & Mata verde & Mata barrosa \\
\hline MATORRAL DE MATA BARROSA & 85,5 & 113 & 1582 & Notro & Mixto C/N & Cohigual \\
\hline MATORRAL DE MATA GUANACO & 98 & 128 & 1792 & Cohigual & Cohigual & Parabrezal \\
\hline LENGAR & 99,36 & 175,26 & 1505 & Mata barrosa & Mata guanaco & Lenga \\
\hline BOSQUE MIXTO COHIGUAL-LENGAR & 93,6 & 166,1 & 2159 & Tundra & Notro & Tundra \\
\hline BOSQUE MIXTO COHIGUAL-NIIRRE & 97,2 & 135,7 & 1357 & Turbera & Mata barrosa & Turbera \\
\hline COHIGUAL DE MAGALANES & 85,6 & 129,85 & 1515 & Parabrezal & Turbera & Mixto C/N \\
\hline BOSQUE DE NIIRRE & 107,0 & 157,55 & 1659 & Mata verde & Parabrezal & Mata verde \\
\hline
\end{tabular}

Tabla 6. Tabla sintética de valoración y clasificación de las distintas unidades. Elaboración propia. C/Ñ: Cohigual-Nirre; C/L: Cohigual-Lengar.

En primer lugar y atendiendo a las tres mata verde con sólo 63,8 puntos. primeras columnas de la Tabla 6 y la Figura 6, se observa un máximo de Con respecto a la suma de los valores puntuación para el INNAT de 107,05 naturales y culturales (INCON), hay puntos, que recae en el bosque de ñirre, unidades que mejoran ostensiblemente seguido del bosque de lenga con casi como la tundra, que pasa a ocupar el 100 puntos, el matorral de mata guanaco con 98 y después unidades forestales como: los bosques mixtos de coigüe de Magallanes-ñirre con 97,2 y de coigüe de Magallanes con lenga 93,6. Por su parte, los últimos puestos en valores naturales serían para la tundra, con 85, 15 puntos, la turbera, con 75 puntos, el parabrezal, con 72,1, y, por último, el matorral de primer puesto con 176,65 puntos. Junto a ella se disponen el bosque de lenga con 175,26 puntos y el bosque mixto de coigüe de Magallanes y lenga con 166, 1 puntos. Los últimos puestos son para la mata barrosa con 113 puntos, la turbera con 97,5 y el parabrezal con 94,6. Estos dos últimos son las únicas unidades que no consiguen llegar a los 100 puntos. 
Con respecto al PRICON, son lógicamente Se observa, también, la abundancia de las tres unidades más amenazadas las que bosques o paisajes próximos al bosque len aparecen como prioritarias a la hora de su su sucesión vegetal) dentro de los primeros conservación, a saber: el bosque mixto de puestos $y$, por lo general, en los tres coigüe-lenga con 2159, ya que, de criterios. No obstante, como matización, si hecho, es de las pocas parcelas que no se bien esto es rigurosamente así en el INNAT, encontraba dentro de un espacio dentro del INCON, como excepción, el protegido con lo que ello supone, el primer puesto lo ocupa la tundra, lo cual matorral de mata guanaco con 1792 habla de sus innegables valores culturales, puntos y el matorral de notro con 1725 mientras que para el PRICON, unidades puntos. En los últimos puestos y, por tanto, como el matorral de mata guanaco y el de con prioridades de conservación relativas notro relegan a otras unidades forestales a bajas nos encontraríamos con la turbera, causa de los riesgos que recaen sobre ellas 1365, el bosque mixto de coigüe y ñirre por sobrepastoreo natural (guanacos) o con 1515 puntos y, por último, el matorral ganadero o por otro tipo de riesgos, como de mata verde con 1238 puntos. incendios y excursionismo, entre otros.

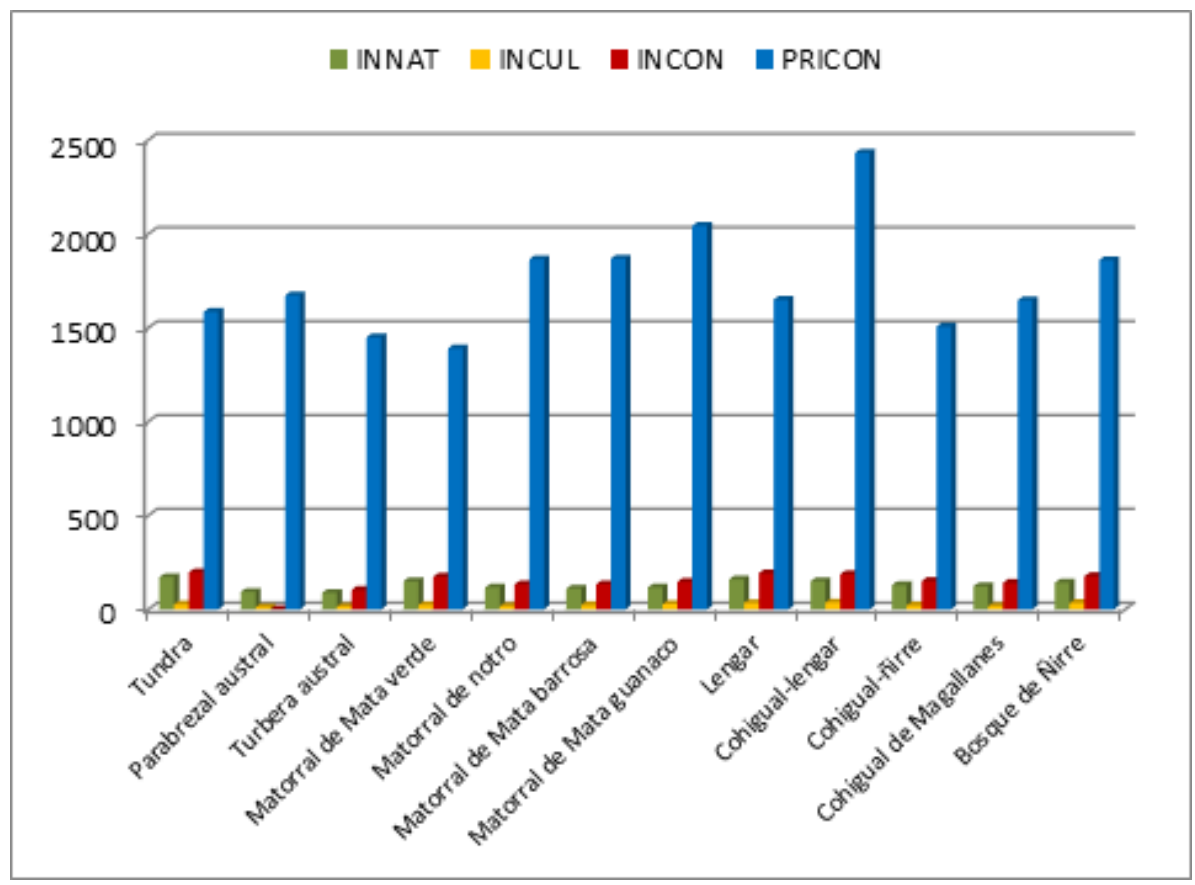

Figura 6. Gráfica de valoración: unidades clasificadas en función del valor del PRICON (elaboración propia).

Sin embargo, los últimos puestos que, en de mata verde muestra las peores general, responden a unidades con valoraciones para el INNAT y el vegetación más rala o de bajo porte, se PRICON, debido a la presencia de usos mantienen, casi de forma homogénea más o menos ancestrales, adquiere dentro de los tres criterios adoptados. No mayor puntuación que otras unidades obstante, mientras el matorral para el INCON. 


\section{DISCUSIÓN}

El inventariado y valoración biogeográfica, a través del método LANBIOEVA, de estos paisajes o formaciones vegetales se ha adaptado perfectamente a las realidades territoriales y ambientales de la Patagonia chilena. En este sentido, tanto el modelo de inventario como los ítems utilizados tradicionalmente para la valoración han servido con alguna salvedad. Por ejemplo, en lo referente a los criterios culturales, se han tenido en cuenta elementos etnográficos, históricos, consuetudinarios, etc., diferentes a los establecidos para otros ámbitos donde se había aplicado la metodología general. También se han debido adaptar los ítems relacionados con el valor patrimonial a través de encuestas sobre el terreno e investigación de los usos tradicionales de la población sobre las diferentes especies. En cualquier caso, a la vista de los datos obtenidos pensamos que la metodología es robusta también para este sector y paisajes.

El número de especies derivadas de los inventarios se encuentra en unas cifras inferiores a las registradas en los sectores mediterráneos de Chile, La Península ibérica o la $P$. balcánica (Lozano et al., $2015 a$ ), pero superiores a los registrados en latitudes altas de Finlandia o Noruega (Lozano et al., 2015b). Hay bastantes especies introducidas, sobre todo en lo referente a las herbáceas, lo cual demuestra la intensa influencia antrópica que han sufrido estos paisajes y ecosistemas. No obstante, dadas las condiciones ambientales, los datos de diversidad de especies son bastante buenos, especialmente los del grupo de matas y trepadoras que con 22 taxones diferentes muestran, en principio la mayor cantidad recogida hasta la fecha (Lozano et al., 2020l pero, por otro lado, demuestran la gran presión antrópica que estos espacios han recibido hasta la fecha. Muchas de ellas se insertan en formaciones o paisajes impactados, seguramente explotados forestalmente y en recuperación vegetativa. No obstante, la carga ganadera excesiva puede ser un impedimento para su total recuperación o para dar lugar a paisajes forestales.

Al igual que ocurre en el ámbito mediterráneo chileno, el número de plantas raras o endémicas es ciertamente alto (Garay y Guineo, 2003). A expensas de poner en marcha esta metodología en islas, lo cierto es que estos dos ámbitos se configuran como aquellos que han registrado valores más altos dentro del grupo de los criterios territoriales (Lozano et al., 2020). Esto demuestra el gran valor de estos paisajes como reservorios de plantas escasas, endémicas, finícolas y, en menor medida, relictas.

El grupo de criterios mesológicos da lugar a puntuaciones muy elevadas para las formaciones boscosas. Éstas generan unas condiciones ecosistémicas esenciales para evitar procesos de erosión y pérdida de suelos así como buenas condiciones bioclimáticas, hidrológicas y, sobre todo, faunísticas. Se configuran como parches forestales esenciales para un buen número de vertebrados e invertebrados forestales (Marín, 2004). No obstante, algunos paisajes de matorral como el de mata guanaco o el de mata barrosa dan lugar a buenos registros y están evitando, en gran medida, importantes procesos de erosión y pérdida de potencial ecológico de ciertos sectores. 
Los valores patrimoniales no son excesivamente elevados. En estos siglos y años se ha perdido, seguramente, un importante bagaje en materia de utilización tradicional de ciertas especies, elementos etnográficos, etc. En este sentido, la información sobre estos y otros aspectos es escasa para este ámbito (Quintanilla, 1989).

Aunque existen paisajes como la turbera y el parabrezal austral que no cuentan con un INCON excesivamente elevado, las amenazas que soportan al encontrarse con ámbitos donde las presiones antrópicas son altas, les otorga un PRICON y, por tanto, una prioridad para su conservación, relativamente alto, de manera que el tomador de decisiones debería tener en cuenta la necesidad de proteger determinados paisajes poco valorados a través, por ejemplo, de nuevas figuras como microreservas que garanticen que estas formaciones modestas y dentro de sectores muy impactados puedan seguir conservándose y gestionarse de manera adecuada (Laguna,

\section{CONCLUSIONES}

A la vista de los objetivos de la investigación planteada, de la disponibilidad en días y de la inclusión de este artículo dentro de un marco más amplio que nos lleva a valorar sistemáticamente unidades o paisajes de latitudes medias y altas, se diseñó un transecto que recogiera la mayor cantidad de unidades dentro de la Patagonia chilena.

En la medida de las posibilidades se ha tendido a tomar parcelas de inventariado y valoración dentro de espacios protegidos de la Península Brunswick y las Torres del

Paine. Se diferenciaron, inventariaron y valoraron doce unidades o paisajes diferentes. Dentro de ellos siete fueron valorados a través de un solo inventario por su homogeneidad mientras que cinco recibieron valoraciones medias a partir de diferentes inventarios derivados de distintas facies dentro de la misma unidad.

En cuanto a la valoración global, indicada por el PRICON, son las tres unidades más amenazadas las que aparecen como preferenciales a la hora de su conservación, a saber: el bosque mixto de coigüe-lenga con 2159 puntos, el matorral de mata guanaco con 1792 puntos y el matorral de notro con 1725 puntos. Todas estas puntuaciones parciales o totales podrán ser tenidas en cuenta a la hora de proteger, ordenar y gestionar estos paisajes y territorios.

Los datos obtenidos, tanto a través del inventariado como de la valoración, muestran un valor incuestionable al circunscribirse dentro de un territorio donde no existen demasiados estudios de este cariz.

Se demuestra la idoneidad de este método LANBIOEVA de valoración para comunidades vegetales del Cono Sur americano, muy distantes espacial y florísticamente de aquellas para las que fue pensado y diseñado en origen, lo que le confiere una plasticidad evaluativa que abarca a muchos de los tipos de vegetación presentes a escala mundial, al menos para aquellas formaciones de ámbitos tropicales, templados y fríos. Fisionómicamente las comunidades de la Patagonia chilena no son tan diferentes de algunas del hemisferio norte donde este sistema ya ha sido ensayado con buenos resultados. 


\section{REFERENCIAS}

Botequilha-Leitao, A. y Ahern, J. (2002). Applying landscape ecological concepts and metrics in sustainable landscape planning. Landscape and Urban Planning 59(2), 65-93. http://dx.doi.org/10.1016/S0169-2046 (02)00005-1

Cadiñaños, J.A. y Meaza, G. (1998a). Bases para una Biogeografía aplicada. Criterios y sistemas de valoración de la vegetación. Geoforma.

Cadiñaños, J.A. y Meaza, G. (1998b). Nueva propuesta metodológica de valoración del interés y de la prioridad de conservación de la vegetación. Actas del Coloquio Internacional de Botánica Pirenaico-Cantábrica.

Cadiñaños, J.A., Meaza, G. y Lozano, P.J. (2002). Valoración del interés y de la prioridad de conservación de bosques y comunidades preforestales de Larra (Alto Pirineo Navarro). La Biogeografía: ciencia geográfica y ciencia biológica. Actas del II Congreso Español de Biogeografía. Universidad de La Laguna.

Cadiñaños, J.A., Lozano, P.J., Meaza, G., Peralta, J., Ollero, A., Díaz, E., lbisate, A., Ormaetexea, O. (2002). Aplicación de una metodología de valoración de la vegetación a riberas fluviales: ensayo en el río Butrón (Bizkaia). Aportaciones geográficas en memoria del Prof. L. Miguel Yetano Ruiz lpp 65-88). Servicio de Publicaciones de la Universidad de Zaragoza.

Chan, K.M.A., Balvanera, P., Benessaia, K., Chapman, M., Díaz, S., Gómez-Baggetum, E.., Gould, R., Hannahs, N., Jax, K., Klain, S., Luck, G.W., Martín-López, B., Muraca,
B., Norton, B., Ott, K., Pascual, U., Satterfield, T., Tadaki, M., Taggart, J. y Turner, N. (2016). Opinion: Why protect nature? Rethinking values and the environment. PNAS $113(6), 1462-1465$ https://doi.org/10.1073/pnas. 15250 02113

Faith, D.P. y Walker, P.A. (1996). Integrating conservation and development: effective trade-offs between biodiversity and cost in the selection of protected areas. Biodiversity and Conservation 5, 43 1-446. https://doi.org/10.1007/BF00056389

Garay, G. y Guineo, O. (2003). Fauna, flora y montaña de Torres del Paine. Ediciones La Prensa Austral.

Guerrido, C. y Fernández, D. (2007). Flora de la Patagonia. Guía para la identificación de plantas y sus hábitats. Editorial Fantástico Sur.

Hoffmann, A., Kalin, M., Liberona, F. Munoz, M., Watson, J. (1998). Plantas altoandinas en la flora silvestre de Chile. Ediciones Fundación Claudio Gay.

Laguna, E. (2014). Origin, concept and evolution of plant micro-reserves: the pilot network of the Valencian Community (Spain). En V. Vladimirov (Ed.), A pilot network of small protected sites for conservation of rare plants in Bulgaria, (chapter 2, 14-24). Institute of Biodiversity and Ecosystem Research y Ministry of Environment and Water.

Lozano, P.J. (2008). Bases para una zoogeografía aplicada. Diseño de una metodología geográfica de atlas de fauna. Aplicación a los vertebrados del sector nororiental de Guipúzcoa. (Tesis Doctoral). 
Lozano, P.J., Cadiñanos, J.A., Longares, L.A. y Meaza, G. (1993). Metodología de evaluación Cid, M.A. (2007). Valoración Biogeográfica del interés naturalístico y de protección de de los tipos de bosque en la combe de unidades de vegetación. Actas XIII Congreso Huidobro (Parque Natural de las Hoces del AGE, 21 1-216.

Ebro-Burgos). Actas del $4^{\circ}$ Congreso Español de Biogeografía, 19.

Lozano, P.J., Cadiñanos, J.A.; Latasa, I.; Quintanilla, V. y Meaza, G. (2015a). Caracterización, valoración y evaluación de los paisajes vegetales de Chile Mediterráneo. Boletín de la AGE 67, 83-103.

Lozano, P.J., Cadiñanos, J.A., Lozano, M.A., Latasa, I., Meaza, G. y Martí, C. (2015b). Aplicación del método de valoración biogeográfico (LANBIOEVA) a ecosistemas del norte de Europa. Eria 97, 189-202.

Lozano, P.J., Varela, R.; Latasa, I.; Lozano-Fernández, A. y Meaza, G. (2020). Biogeographic assessment of vegetal landascape, at a global scale, trough "LANBIOEVA" methodology (Landscape Biogeographical Evaluation). XXXIV UGI Congress Book, 15.

Luebert, F. y Pliscoff, P. (2006). Sinopsis bioclimática y vegetacional de Chile. Editorial Universitaria S.A.

Marín, M. (2004). Lista comentada de las aves de Chile. Lynx Ediciones.

McNeill, J.R. (2003). Algo nuevo bajo el sol. Historia medioambiental del mundo en el siglo XX. Alianza Editorial.

Meaza, G. y Ormaetxea, O. (1992). Propuesta metodológica de valoración fitogeográfica de unidades de paisaje vegetal. Homenaje al investigador Félix $M$. Ugarte Elorza, 369-391.

Meaza, G. (1994). Valoración de comunidades preforestales semidecíduas de neblisilva y pluvisilva centroamericanas. Boletín América Latina -AGE 14, 2-13.

Meaza, G., Cadiñanos, J.A. y Lozano, P.J. (2006). Valoración biogeográfica de los bosques de la reserva de la biosfera de Urdaibai (Vizcaya). Actas del III Congreso Español de Biogeografía, 399-41 1

Pisano, E. (1977). Fitogeografía de Fuego-Patagonia chilena I. Comunidades vegetales entre las latitudes $52^{\circ}$ y $56^{\circ} \mathrm{S}$. Anales del Instituto de la Patagonia 8, $121-250$.

Quintanilla, V.G. (1989). Fitogeografía y Cartografía Vegetal de Chile Austral. Contribuciones Cientificas y Tecnológicas; Área Geociencias VII. Universidad de Santiago de Chile.

Rodríguez, R., Ruiz, E. y Elissetche, J.P. (2005). Árboles en Chile. Universidad de Concepción.

Strijker, D., Siitsma, F.J. y Wiersma, D. (2000). Evaluation of nature conservation: An application to the Dutch Ecological Network. Environmental and Resource Economics 16, 363-378.

Tscharntke, T., Steffan-Dewenter, I., Kruess, A. y Thies, C. (2002). Contribution of small hábitat fragments to conservation of insect communities of Grassland-Cropland Landscapes. Ecologocal Applications 12(2), 354-363.

Wilson, E.O. (1994): La diversidad de la vida. Editorial Crítica. 